\title{
Influência de medicamentos no risco de queda em idosos
}

\author{
Influence of medicines on the risk of falls in the elderly \\ Influencia de los medicamentos em el riesgo de caídas em los ancianos
}

Isadora de Figueiredo Infante Falleiros ${ }^{1}$, Malu Labecca Selicani ${ }^{1}$, Mauro Lúcio Alves da Costa ${ }^{1}$, Rita de Cássia Chaves Garcia Barbosa ${ }^{1}$, Gérsika Bitencourt Santos ${ }^{1 *}$.

\begin{abstract}
RESUMO
Objetivo: Revisar a influência de medicamentos no risco de queda em pacientes idosos. Métodos: Trata-se de uma revisão de literatura que teve como busca bibliográfica as bases de dados National Library of Medicine (PubMED), Scientific Electronic Library Online (SciELO), Literatura Latino-americana e do Caribe em Ciências da Saúde (LILACS) e Google Acadêmico. Foram utilizados artigos publicados entre os anos de 2002 a 2021 que tivessem relação com o uso de medicamentos e o risco de queda em idosos. Resultados: Dentre os 21 artigos selecionados para a revisão de literatura, todos eles demonstram que o risco de queda é aumentado em idosos que utilizam medicamentos, dentre eles os benzodiazepínicos e anti-hipertensivos. Considerações Finais: Os idosos que utilizam algumas classes de medicamentos têm um aumentado de risco de queda se comparados com indivíduos da mesma idade que não utilizam tais medicamentos. Portanto, é de extrema relevância a prescrição correta de medicamentos para evitar os danos causados pelas quedas nos indivíduos da terceira idade.
\end{abstract}

Palavras-chave: Acidentes por quedas, Saúde do idoso, Avaliação de medicamentos.

\section{RESUME}

Objective: Review of influence of drugs without risk of falling in elderly patients. Methods: It is an integrative literature review that had as bibliographic search as databases National Library of Medicine (PubMED), Scientific Electronic Library Online (SciELO), Latin American and Caribbean Literature in Health Sciences (LILACS) and Google Scholar, articles published between 2002 and 2021 that were related to the use of medications and the risk of falling in the elderly. Results: Among the 21 articles selected for the literature review, all of them demonstrate that the risk of falling is aged in the elderly who use it, among them benzodiazepines and antihypertensives. Final Considerations: The elderly who use medications have an increased risk of falling compared to individuals of the same age who do not use medications. Therefore, the correct prescription of medications is extremely important to avoid the damage caused by falls in the elderly.

Key words: Accidents due to falls, Health of the elderly, Assessment of medication.

\section{RESUMEN}

Objetivo: Revisar la influencia de los fármacos sobre el riesgo de caídas en pacientes ancianos. Métodos: Es una revisión de literatura integradora que tuvo como búsqueda bibliográfica como bases de datos Biblioteca Nacional de Medicina (PubMED), Biblioteca Electrónica Científica en Línea (SciELO), Literatura Latinoamericana y del Caribe en Ciencias de la Salud (LILACS) y Google Scholar, artículos publicados entre 2002 y 2021 que estaban relacionados con el uso de medicamentos y el riesgo de caídas en los ancianos. Resultados: De los 21 artículos seleccionados para la revisión de la literatura, todos demuestran que el riesgo

1 Universidade José do Rosário Vellano (UNIFENAS), Alfenas - MG. *E-mail: gersika.santos@unifenas.br 
de caídas es la edad en los ancianos que lo consumen, entre ellos las benzodiazepinas y los antihipertensivos. Consideraciones Finales: Los ancianos que usan medicamentos tienen un mayor riesgo de sufrir caídas en comparación con las personas de la misma edad que no usan medicamentos. Por tanto, la correcta prescripción de los medicamentos es de suma importancia para evitar los daños que provocan las caídas en los ancianos.

Palabras clave: Accidentes por caídas, Salud de las personas mayores, Evaluación de medicamentos.

\section{INTRODUÇÃO}

Sabe-se que nas últimas décadas houve um declínio da taxa de fertilidade combinado com um aumento da expectativa de vida, levando a um crescimento da população idosa mundialmente, incluindo o Brasil. Estudos do Instituto Brasileiro de Geografia e Estatística (IBGE), por meio da Pesquisa Nacional por Amostra de Domicílios (PNAD), apontam que a população idosa em 2017 era de $14,6 \%$ enquanto em 2010 era de 10,8\%, além disso o IBGE projeta que em 2060 aproximadamente $1 / 3$ da população brasileira será de pessoas idosas (MINISTÉRIO DA CIDADANIA, 2018).

Diante dessa nova vertente é importante ressaltar que a Organização Mundial da Saúde (OMS) (2005), define idoso como: indivíduos que apresentam 60 anos de idade ou mais em casos de países subdesenvolvidos, como é o caso do Brasil, e para países desenvolvidos aqueles com mais de 65 anos de idade, sendo importante salientar que essa definição foi estabelecida pela primeira vez pela Organização das Nações Unidas, por meio da Resolução 39/125, durante a Primeira Assembleia Mundial das Nações Unidas sobre o Envelhecimento da População (SANTOS SSC, 2010).

Estudos realizados em um serviço de ortopedia e traumatologia demonstraram que $72,05 \%$ dos idosos admitidos com fratura faziam uso de algum tipo de medicamento. Isso se dá devido ao fato de que com o avanço da idade os índices de doenças neurodegenerativas, psiquiátricas, cardiovasculares e metabólicas aumentam proporcionalmente, tendo como consequência um aumento das medicações usadas. Além disso, uma outra vertente que soma ao uso isolado ou associado de alguns medicamentos relacionado ao risco de queda é a redução da massa muscular e óssea em conjunto com a perda de equilíbrio patológica no idoso. Com isso, podemos concluir que o risco de queda está diretamente relacionado não só às alterações fisiopatológicas já esperadas com o avanço da idade, mas também com o uso de determinados medicamentos e a fatores externos ambientais (HAMARA A, et al., 2007).

É importante salientar que há fatores intrínsecos e extrínsecos que predispõem ao risco de queda em idosos. Em primeira instância, estudos realizados na cidade de Porto Alegre demonstram que o avanço da idade associado aos fatores intrínsecos, isto é, do próprio sujeito, como percepção postural, equilíbrio e hipotensão postural somados aos fatores extrínsecos como moradia, iluminação e deambulação dos pacientes, contribuem para o risco de queda na terceira idade. Observou-se que indivíduos que sofreram quedas que resultaram em fratura, desenvolveram limitações após o tratamento, seja ele cirúrgico ou conservador (ALMEIDA ST, et al., 2012).

Como dito, o aumento do índice de queda em idosos está diretamente relacionado a fatores intrínsecos como alterações posturais devido a modificações no sistema sensorial e efetor. Partindo do sistema sensorial encontramos alterações visuais visto que com o avanço da idade temos um depósito de lipídeos na córnea como também uma redução do tamanho da pupila diminuindo a acuidade visual e visão periférica, somada a alteração visual temos um distúrbio do sistema vestibular que tem como objetivo promover ajustes oculares conforme os movimentos da cabeça, no idoso iremos deparar com uma redução de células nesse sistema resultando em falhas na transmissão de posicionamento levando a um quadro de desequilíbrio (MEIRELES AE, et al., 2010).

Em contrapartida o sistema efetor tem como pilar o sistema musculoesquelético onde encontraremos uma redução do líquido sinovial, adelgaçamento das cartilagens, encurtamento e rigidez de ligamentos, promovendo uma redução da amplitude de movimento (RIBEIRO LCC, et al., 2009). Em síntese, é possível 
observar que o próprio ato de envelhecer está associado a um risco de queda que quando associado ao uso de medicações aumenta ainda mais, visto que estas podem atuar de maneira direta no sistema sensorial e efetor.

Pesquisas demonstram que as pacientes idosas têm maior predisposição ao risco de queda. Tal fato pode ser justificado por algumas vertentes, como a das alterações metabólicas/endócrinas que acontecem após a menopausa. Isso se deve ao fato que após a menopausa ocorre uma diminuição do nível de estrogênio circulante, um dos hormônios que está relacionado aos mecanismos de reabsorção óssea, inibindo a atividade de osteoclastos. Perante tais alterações, pacientes idosas têm maior predisposição a quedas quando comparamos com indivíduos do sexo masculino com a mesma idade. Outrossim, nota-se que o uso de medicamentos por essa população pode influenciar e aumentar ainda mais o risco de queda, uma vez que podem causar hipotensão postural e sedação (GARCIA BP, 2018).

A medicação, até ser prescrita para o idoso, envolve algumas etapas: elaboração, prescrição, administração e acompanhamento clínico. Devido à complexidade desse fato, associado a temática de que o idoso já é um paciente polimedicado, predispõe à iatrogenias. O médico, por sua vez, precisa conhecer os medicamentos potencialmente inapropriados (MIPs) fármacos que têm maior capacidade de causar efeitos colaterais do que benefício para o paciente que faz uso de tal medicação. Dentre essas drogas, pode-se citar alguns anti-hipertensivos, anti-histamínicos, benzodiazepínicos e anticonvulsivantes. Portanto, é de extrema importância que alguns medicamentos dessas classes acima não sejam prescritos aos idosos para que evitemos o risco de efeitos colaterais (GORZONI MR, et al., 2012).

Com isso, foram realizados inúmeros estudos em literaturas sistemáticas, cujas conclusões se demonstraram heterogêneas. Embora houvesse divergências entre as conclusões, foi evidenciado a necessidade de adquirir mais conhecimento e de realizar mais estudos sobre a prevalência e a distribuição dos fatores que contribuem para a ocorrência de queda em idosos. Devido a tal motivo, é de extrema importância a investigação das buscas por assistências à saúde entre as pessoas da terceira idade, como meio de analisar os indicadores de gravidades relacionados às quedas. Portanto, o objetivo dessa revisão de literatura é analisar a influência dos medicamentos no risco de quedas e fraturas em idosos, mostrando as principais classes de fármacos que contribuem para tal evento e evitar que essa moléstia continue persistindo na terceira idade (AMORIM JSC, et al. 2021).

Embora as quedas sejam consideradas um evento normal na terceira idade, constitui um problema de saúde pública pois, além de fraturas, a qualidade de vida e o desejo de realizar tarefas que antes o idoso realizava são diminuídos (REZENDE CP, et al., 2012). Por isso, o estudo objetivou revisar a influência de medicamentos no risco de queda em pacientes idosos.

\section{MÉTODOS}

Os artigos utilizados nesta revisão bibliográfica integrativa foram pesquisados nas bases de dados National Library of Medicine (PubMED), Scientific Electronic Library Online (SciELO), Literatura Latino-americana e do Caribe em Ciências da Saúde (LILACS) e Google Acadêmico, publicados entre os anos de 2002 a 2021. Os descritores utilizados na busca foram: "acidentes por quedas"; "saúde do idoso"; "medicamentos" "risco de queda em idosos";

Em relação aos critérios de inclusão, foram selecionados os artigos que apresentavam dados originais e secundários sobre a associação entre risco de queda em idosos, junto com correlações medicamentosas. Quanto aos critérios de exclusão, foram desconsiderados trabalhos em duplicidade, publicados anteriormente ao ano de 2002 e aqueles que, apesar de possuírem os descritores elegidos, não abordavam a temática da pesquisa diretamente. Após a aplicação dos critérios, foram lidos 90 resumos; analisados 48 artigos completos e selecionados 24 artigos para a inclusão nesta revisão, conforme demonstrado na Tabela 1. 
Tabela 1 - Distribuição das referências bibliográficas obtidas nas bases de dados Lilacs, Pubmed, Scielo e Google Scholar, segundo as palavras chaves selecionadas.

\begin{tabular}{|c|c|c|c|c|c|}
\hline $\begin{array}{l}\text { Bases } \\
\text { de } \\
\text { Dados }\end{array}$ & $\begin{array}{l}\text { Palavra-chave Cruzadas } \\
\text { Concomitantemente } \\
\text { (como descritores e } \\
\text { palavras do resumo) }\end{array}$ & $\begin{array}{l}\text { Número de } \\
\text { referências } \\
\text { obtidas }\end{array}$ & $\begin{array}{l}\text { Resumos } \\
\text { Analisados }\end{array}$ & $\begin{array}{l}\text { Referências } \\
\text { Selecionadas } \\
\text { para Análise }\end{array}$ & $\begin{array}{l}\text { Selecionados } \\
\text { para a Revisão }\end{array}$ \\
\hline \multirow{5}{*}{ Lilacs } & Acidente por queda & 169 & 12 & 6 & 2 \\
\hline & $\begin{array}{l}\text { Acidente por queda, } \\
\text { Fatores de risco }\end{array}$ & 37 & 2 & 2 & 0 \\
\hline & Polifarmácia, Idosos & 225 & 3 & 1 & 0 \\
\hline & $\begin{array}{l}\text { Acidente por queda, } \\
\text { medicamentos }\end{array}$ & 3 & 3 & 2 & 0 \\
\hline & $\begin{array}{c}\text { Uso de medicamento em } \\
\text { idosos AND acidente por } \\
\text { queda }\end{array}$ & 20 & 7 & 3 & 2 \\
\hline \multirow{4}{*}{ PubMed } & Medicamentos, queda & 3 & 3 & 2 & 1 \\
\hline & Idosos, medicamentos & 49 & 4 & 3 & 1 \\
\hline & $\begin{array}{c}\text { Medicamentos, idoso, } \\
\text { queda }\end{array}$ & 3 & 3 & 2 & 1 \\
\hline & Idoso, queda & 55 & 9 & 6 & 4 \\
\hline \multirow{4}{*}{ SciELO } & $\begin{array}{l}\text { Acidentes por quedas, } \\
\text { idosos }\end{array}$ & 152 & 5 & 2 & 1 \\
\hline & $\begin{array}{l}\text { Medicamento, idoso, } \\
\text { queda }\end{array}$ & 56 & 6 & 4 & 1 \\
\hline & $\begin{array}{c}\text { Medicamentos, risco que } \\
\text { queda }\end{array}$ & 45 & 9 & 5 & 1 \\
\hline & Idoso, queda & 252 & 5 & 3 & 1 \\
\hline \multirow{4}{*}{$\begin{array}{l}\text { Google } \\
\text { Scholar }\end{array}$} & $\begin{array}{l}\text { Acidente por queda, } \\
\text { idosos }\end{array}$ & 15.500 & 8 & 2 & 1 \\
\hline & $\begin{array}{l}\text { Medicamento, idoso, } \\
\text { queda }\end{array}$ & 15.300 & 1 & 1 & 0 \\
\hline & Idosos, medicamentos & 16.700 & 3 & 2 & 1 \\
\hline & Idoso, queda & 15.300 & 7 & 2 & 1 \\
\hline
\end{tabular}

Fonte: Falleiros IFI, et al., 2021.

\section{RESULTADOS}

Dentre os dezoito artigos analisados, publicados entre os anos de 2002 e 2021, todos correlacionam diretamente o uso de medicamentos com o risco aumentado de queda em idosos (Quadro 1). Dessa maneira, se solidifica o tema como de relevância mundial, uma vez que, baseado em estudos caso-controle, revisões de literaturas, artigos originais e relatos clínicos, se prova essa correlação direta a nível equivalente entre a população idosa. 
Quadro 1 - Distribuição das referências incluídas na revisão integrativa, de acordo com o ano de publicação e autor.

\begin{tabular}{|c|c|c|}
\hline $\mathbf{N}^{\circ}$ & Autores (Ano) & Principais Achados \\
\hline 1 & SILVIA IGP, et al. (2019) & $\begin{array}{l}\text { Trata-se de um estudo transversal não probabilístico de abordagem quantitativa. Tal estudo tem } \\
\text { como objetivo analisar o perfil sociodemográfico e clínico de idosos quanto ao risco de queda, } \\
\text { tendo como principal achado uma vulnerabilidade de idosos para quedas maiores no sexo } \\
\text { feminino como também para aqueles que faziam uso de medicamentos e possuíam pele menos } \\
\text { uma comorbidade. }\end{array}$ \\
\hline 2 & OLIVEIRA T, et al. (2018) & $\begin{array}{l}\text { Trata-se de um estudo descritivo, exploratório, transversal e quantitativo realizado com } 31 \\
\text { idosos em uma Unidade de Cuidados na Comunidade. Tal estudo visa constatar quais são os } \\
\text { principais fatores de risco relacionado com a queda de pacientes idosos, tendo como achado } \\
\text { as alterações fisiopatologias advindas com a idade como também o uso de benzodiazepínicos, } \\
\text { polimedicação e influência do ambiente externo. }\end{array}$ \\
\hline 3 & LUIZ IC e BRUM AKR (2017) & $\begin{array}{l}\text { Trata-se de um estudo descritivo de abordagem quantitativo realizado com } 36 \text { idosos. Tal estudo } \\
\text { tem como objetivo associar os fatores intrínsecos de queda em idosos em ambiente domiciliar } \\
\text { em idosos com doenças crônicas, tendo como fatores mais prevalentes o uso de medicamentos, } \\
\text { principalmente os hipotensores. }\end{array}$ \\
\hline 4 & GARCÍA BP, et al. (2018) & $\begin{array}{l}\text { Trata-se de um estudo transversal descritivo utilizando uma amostra aleatória. Tal estudo tem } \\
\text { como objetivo determinar qual o número de medicamentos consumidos diariamente está } \\
\text { influenciando risco de queda em idosos, tendo como achado que o consumo de quatro } \\
\text { medicamentos ou mais é um fator de rico para quedas. }\end{array}$ \\
\hline 5 & MOREIRA FSM, et al. (2020) & $\begin{array}{l}\text { Trata-se de um estudo transversal que visa averiguar a prevalência do uso de medicamentos } \\
\text { potencialmente inapropriados (MPI) e os fatores a eles associados. Tal estudo teve como } \\
\text { principal achado os benzodiazepínicos que são uma classe de MPI ainda muito prescrita para } \\
\text { idosos, tendo como consequência direta o aumento do risco de queda dessa população. }\end{array}$ \\
\hline 6 & SOUZA LMM, et al. (2017) & $\begin{array}{l}\text { Trata-se de uma revisão sistemática da literatura baseada em plataformas digitais com o } \\
\text { objetivo de identificar os fatores de risco para quedas em idosos. Tal estudo teve como achado } \\
\text { que a polimedicação, os agentes anti-hipertensivos e os benzodiazepínicos são fatores de risco } \\
\text { para quedas em idosos. }\end{array}$ \\
\hline
\end{tabular}




\begin{tabular}{|c|c|c|}
\hline $\mathbf{N}^{\circ}$ & Autores (Ano) & Principais Achados \\
\hline 7 & NETO AHA, et al. (2017) & $\begin{array}{l}\text { Trata-se de um estudo transversal realizado com } 45 \text { idosos em Instituições de Longa } \\
\text { Permanência para Idosos de João Pessoa, Brasil, nos meses de junho e julho de } 2016 . \\
\text { Concluiu-se que } 66 \% \text { dos indivíduos já apresentaram quedas e que um dos fatores para tal } \\
\text { problema são as medicações usadas no tratamento da hipertensão, visto que podem causar } \\
\text { hipotensão postural. }\end{array}$ \\
\hline 8 & SANTOS SSC, et al. (2012) & $\begin{array}{l}\text { Trata-se de uma revisão integrativa de literatura que, frente às possíveis implicações e } \\
\text { consequências das quedas na vida dos idosos e sua relação com o cuidado dispensado pelo } \\
\text { enfermeiro, apresentou-se como questão de pesquisa: a partir do diagnóstico de enfermagem } \\
\text { da North American NursingDiagnosisAssociation Risco de quedas, que fatores podem ser } \\
\text { identificados na população idosa, contida na literatura científica brasileira e estrangeira, no } \\
\text { período de } 2005 \text { a } 2010 \text {. Foram identificados } 4 \text { tipos de fatores que contribuem para quedas, } \\
\text { sendo eles: ambiental, cognitivo, fisiológico e neoplasia. Concluiu-se que os riscos para quedas } \\
\text { relacionados ao uso de medicamentos revelaram que a utilização contínua de diversos } \\
\text { fármacos predispõe o idoso a reações adversas e a maiores interações medicamentosas, o que } \\
\text { foi preponderante no risco de cair. }\end{array}$ \\
\hline 9 & COUTINHO ESF e SILVA SD (2002) & $\begin{array}{l}\text { Foi realizado um estudo caso-controle sobre o uso de determinados medicamentos como fator } \\
\text { de risco para hospitalização por fratura após queda em indivíduos com } 60 \text { anos ou mais na } \\
\text { cidade do Rio de Janeiro, Brasil. Concluiu-se que o aumento do risco de queda está associado } \\
\text { ao uso de bloqueadores de canal de cálcio e benzodiazepínicos. }\end{array}$ \\
\hline 10 & RODRIGUES MCS e OLIVEIRA C (2016) & $\begin{array}{l}\text { Trata-se de uma revisão integrativa dos estudos publicados no período de janeiro de } 2008 \text { a } \\
\text { dezembro de } 2013 \text { nas plataformas MEDLINE e EMBASE com o objetivo de identificar e } \\
\text { sintetizar estudos que examinam as interações medicamentosas (IM) e reações adversas a } \\
\text { medicamentos (RAM) em idosos polimedicados. }\end{array}$ \\
\hline 11 & REZENDE CP, et al. (2012) & $\begin{array}{l}\text { Trata-se de uma revisão de literatura sistemática sobre os estudos em relação aos } \\
\text { medicamentos associados ao risco de quedas. Não houve preocupações com limites de data } \\
\text { de publicações. Portanto, conclui-se que, certos medicamentos fazem realmente parte dos } \\
\text { fatores de risco para as quedas em idosos, chamando a atenção, no artigo em questão, para } \\
\text { os benzodiazepínicos e diuréticos. }\end{array}$ \\
\hline
\end{tabular}




\begin{tabular}{|c|c|c|}
\hline $\mathbf{N}^{\circ}$ & Autores (Ano) & Principais Achados \\
\hline 12 & NASCIMENTO JS e TAVARES DMS (2016) & $\begin{array}{l}\text { Trata- se de um estudo de abordagem quantitativa, tendo como objetivo determinar a frequência } \\
\text { de quedas em idosos nos últimos } 12 \text { meses (em relação a data da revisão em questão) } \\
\text { comparando, assim, a gama sociodemografica, clínicas, de saúde e capacidade funcionais } \\
\text { entre aqueles que sofreram por quedas e outros que não. Conclui-se que, é nítido é prevalência } \\
\text { das quedas em idosos que moram na zona urbana, de sexo feminino e com } 80 \text { anos ou mais. }\end{array}$ \\
\hline 13 & HAMARA A, et al. (2007) & $\begin{array}{l}\text { O artigo em questão se trata de um estudo de caso-controle. Foi comparado, no ano de } 2005 \text {, } \\
205 \text { idosos que possuíam fraturas com outros } 205 \text { que estavam completamente hígidos, } \\
\text { comparando estatisticamente e analisando os possíveis causadores dessas quedas e fraturas } \\
\text { decorrentes de quedas. Concluiu-se que, foi significante a diferença entre os grupos controle, } \\
\text { podendo dizer, então, que o uso prévio de medicamentos é um importante fator de risco, } \\
\text { principalmente as drogas que alteram o grau de atenção, pressão arterial e respostas motoras. }\end{array}$ \\
\hline 14 & ALVES RLT, et al. (2017) & $\begin{array}{l}\text { O artigo se trata de um estudo de corte transversal, cujo objetivo foi investigar a incidência de } \\
\text { quedas em idosos na cidade de Barbacena-MG, analisando os fatores causais e as } \\
\text { consequências. Sendo assim, foi feito questionários, avaliando a presença ou não de } \\
\text { comorbidades, uso de medicamentos e fatores ambientais, como certos moveis em ambiente } \\
\text { domiciliar que pode acabar causando alguma queda, como degraus e tapetes, com isso, foi } \\
\text { avaliado também idosos que moram sozinhos e aqueles que possuem alguém como ajudante. } \\
\text { Portanto, teve como conclusão a incidência de e } 36,41 \% \text { de idosos que sofreram quedas, sendo } \\
\text { o fator de risco mais avaliado: Uso de medicamentos. }\end{array}$ \\
\hline 15 & TOMAZ SAG, et al. (2017) & $\begin{array}{l}\text { A pesquisa em questão se trata de um estudo de caráter transversal, descritivo, exploratório e } \\
\text { quantitativo. O objetivo foi analisar quais medicamentos (especificamente entre os } \\
\text { benzodiazepínicos e diuréticos) que influenciam na queda em idosos. Sendo assim, fo } \\
\text { concluído que os benzodiazepínicos são certamente um fator de risco para as quedas, } \\
\text { aumentando as ocorrências das mesmas, principalmente no período da manhã. Já os diuréticos } \\
\text { não tiveram influência significativa das ocorrências de quedas, porém, se faz nítido a } \\
\text { importância de tomar muito cuidado para o seu uso. Portanto, o estudo denotou a necessidade } \\
\text { de uma conscientização dos familiares dos idosos em questão e dos ajudantes, para que fiquem } \\
\text { mais atentos aos possíveis efeitos adversos. }\end{array}$ \\
\hline
\end{tabular}




\begin{tabular}{|c|c|c|}
\hline No & Autores (Ano) & \multicolumn{1}{c|}{ Principais Achados } \\
\hline 16 & AMORIM JSC, et al. (2021) & $\begin{array}{l}\text { Trata-se de um estudo transversal, incluindo 10.537 idosos participantes da Pesquisa Nacional } \\
\text { de Saúde (2013). O objetivo principal foi estimar a prevalência e os fatores associados à queda } \\
\text { grave em idosos brasileiros. Percebeu-se que a prevalência de queda grave foi entre mulheres, } \\
\text { vivendo sem cônjuge, sedentários no lazer, com multimorbidades. Isso se voltou para a } \\
\text { conclusão de que a prevalência de queda grave foi elevada e multifatorial. }\end{array}$ \\
\hline 17 & DEGANI GC, et al. (2014) & $\begin{array}{l}\text { Estudo retrospectivo e exploratório, com a análise de dados secundários de um banco de dados } \\
\text { de um hospital geral terciário. Foram estudados 131 idosos, média de idade 69 anos. Objetivou- } \\
\text { se identificar o perfil sociodemográfico de idosos vítimas de trauma, caracterizar doenças } \\
\text { preexistentes e medicamentos utilizados no domicílio; calcular índices de trauma e desfecho } \\
\text { clínico. Concluiu-se que houve correlação das quedas com o predomínio do uso de } \\
\text { medicamentos em domicílio, principalmente, de anti-hipertensivos, diuréticos e antiagregante } \\
\text { plaquetário/ anticoagulante. }\end{array}$ \\
\hline 18 & DANZA DÁ, et al. (2015) & $\begin{array}{l}\text { Foi realizado um estudo de caso-controle durante seis meses em pacientes com fratura } \\
\text { traumática de quadril. O objetivo foi determinar se há associação entre o consumo de BZD e o } \\
\text { risco de fratura de quadril. "Caso" foi definido como um paciente com fratura traumática de } \\
\text { quadril. Quanto aos resultados, foram obtidos } 29 \text { casos e } 60 \text { controles e o OR para fratura de } \\
\text { quadril em pacientes que consumiram BZD foi de 4,5 (IC 95\% 1,7-11,6). Dessa forma, viu-se } \\
\text { que os BZDs aumentam o risco de fratura de quadril e, ainda, são consumidos por períodos } \\
\text { desaconselhados. }\end{array}$ \\
\hline
\end{tabular}

Fonte: Falleiros IFI, et al., 2021. 
De acordo com o quadro acima, sete artigos remetem o uso de benzodiazepínicos (BZD) como um fator de risco para quedas. Um dos estudos sugere a associação entre o consumo de BZD e a presença de uma fratura de quadril traumática (DANZA DA, et al., 2015). Além disso, alguns estudos também defendem que 0 mecanismo de risco de queda advindo do BZD se concentra na sua ação sedativa, porque causa alterações motoras, além de sua ação bloqueadora de canais alfas, visto que aumenta a probabilidade de hipotensão postural (REZENDE CP, et al., 2012).

Quatro artigos dentre os presentes na tabela ressaltaram a ligação direta dos anti-hipertensivos com a ocorrência de queda em população idosa. Ainda, viu-se que a queda está duplamente ligada com mecanismos circulatórios, um viés indicativo para os anti-hipertensivos e outro, correlacionando diretamente a queda com a própria Hipertensão Arterial Sistêmica (HAS). A Hipertensão é citada porque altera condições respiratórias e hemodinâmicas do paciente, antecedendo estados de hipóxia e hipoperfusão. Pode-se até confundir no momento da avaliação da gravidade após a o acontecimento do trauma, pois a resposta hemodinâmica é limitada nesta população e o valor da pressão arterial apresentada no idoso hipertenso pode ser considerado normal, quando na verdade, já se inicia um quadro de hipovolemia (DEGANI GC ei al., 2014).

Ademais, durante o estudo, observou-se uma prevalência significativa na ocorrência de quedas em pacientes do sexo feminino; fator associado ou não ao uso de medicamentos. Nos estudos, acredita-se que essa prevalência seja multifatorial, englobando de alterações endócrinas e metabólicas pós menopausa, até pela maior exposição das idosas a comportamentos de risco, tais como uso inapropriado de sapatos e preferência pela realização de atividades. Essa associação de quedas ao sexo feminino esteve presente em pesquisas nacionais e internacional (NASCIMENTO JS e TAVARES DMS, 2016).

\section{DISCUSSÃO}

Perante o estudo realizado foi possível perceber que o envelhecimento desassistido é um problema de saúde pública mundial, uma vez que o grau de dependência aumenta tendo em vista as alterações osteomusculares, alterações no equilíbrio e propriocepção que somado ao uso de determinadas medicações tem-se um aumento do risco de queda na população idosa (LUIZ IC e BRUM AKR, 2017).

Com o avanço da idade o uso de medicamentos aumenta relativamente devido não só a maior incidência de doenças crônicas como também devido às alterações fisiopatológicas advindas com a idade. Sabe-se que o processo de envelhecimento altera as funções primordiais dos órgãos e como consequência disso temos alterações na farmacocinética e farmacodinâmica dos fármacos, pensando principalmente na eliminação hepática e excreção renal. Diante desses contextos, tem-se uma lista de fármacos potencialmente inapropriados para idosos dentro dos Critério de Beers, visto que esses fármacos estão associados de maneira direta com o aumento do risco de quedas, fraturas, hospitalização e mortalidade. Mesmo perante a esse cenário a prescrição de medicamentos potencialmente inapropriados (MPI) no Brasil varia de $24,60 \%$ a $82,6 \%$, tendo como principais classes os antipsicóticos, os benzodiazepínicos e a sulfoniuréias. Sendo os benzodiazepínicos, principalmente os de ação prolongada, os mais preocupantes, visto que eles aumentam o efeito sedativo residual e consequentemente aumentam o risco de queda (GARCIA BP, et al., 2018).

Em consonância com a literatura, os estudos selecionados para análise nesta revisão integrativa ressaltam a influência significativa de fármacos hipnóticos e sedativos, como é o caso dos benzodiazepínicos (BNZs), no risco de queda (OLIVEIRA T, et al., 2018). Os BNZs representam uma classe farmacológica cuja ação farmacodinâmica é sinérgica à ação do GABA. De maneira geral, a ação se dá pela abertura de Canais de Cloreto após a ligação do medicamento ao receptor do tipo GABA 1, visto que causa a hiperpolarização do neurônio e sua consequente inibição. Embora muito semelhantes, o mecanismo de ação dos fármacos dessa classe um pouco de diferem. O receptor de GABA é formado por subunidades $\alpha, \beta, \gamma$. Assim, cada BNZ se liga a uma subunidade e produzirá efeitos farmacológicos que podem divergir - a subunidade $\alpha 1$, por exemplo, produz efeito predominante hipnótico; $\beta$, anticonvulsivante, e $\gamma$, relaxante muscular. Portanto, a ação do fármaco a determinado sítio de ligação configura o efeito esperado pela medicação que deve ser somado as alterações do equilíbrio, percepção, tempo de reação e força muscular, tornando evidente que o consumo dessa medicação pode aumentar de 2 a 4 vezes o risco de queda. Além disso, a classe pode ser pilar de 
interações medicamentosas com outras drogas que agem no sistema nervoso central, tornando o seu uso mais alarmante uma vez que a maioria dos idosos fazem uso de 4 ou mais medicamentos, assim apresentando um risco elevado de queda e uma piora de seu estado funcional (SOUZA AR, et al., 2013).

Levando em consideração as doenças crônicas podemos ressaltar a hipertensão como uma das mais prevalentes, sendo necessário o uso de determinados medicamentos para o controle dessa. Sabe-se que o tratamento deve ser individualizado, porém há indicações prévias para o tratamento dessa patologia, sendo mais indicado para pacientes idosos uma monoterapia com alguns medicamentos preferenciais como diuréticos (DIU) tiazídicos, Bloqueadores de Canais de Cálcio (BCC), Inibidores da Enzima Conversora da Angiotensina (IECA) e os Bloqueadores dos Receptores AT1 da Angiotensina II (BRA) (BARROSO WKS, et al., 2021)

Os DIU têm como sítio de atuação o néfron, levando a uma depleção de sódio, gerando uma redução do fluido extracelular e do débito cardíaco, podendo acentuar o risco de queda devido a um distúrbio hidroeletrolítico gerando uma depleção de volume e hipocalemia, levando a uma hipotensão postural (SILVA $P, 2017)$. Em conjunto aos fatores intrínsecos do uso dos DIU devemos somar fatores externos ambientais, uma vez que tal medicação aumenta o número de micções fazendo com que os idosos se levantem mais, aumentando o risco de queda (TOMAZ SAG, et al., 2017). Em contrapartida, um outro estudo aponta um possível efeito protetor dos DIU tiazídicos por reduzirem a excreção urinária de cálcio, aumentando a densidade óssea (HAMARA A, et al., 2007).

Os BCC atuam bloqueando os canais de cálcio na membrana das células presentes na musculatura lisa das arteríolas, reduzindo a quantidade de cálcio presente no interior das células, dificultando a contração muscular, levando a uma vasodilatação e uma redução da resistência vascular periférica. Já os IECA agem bloqueando a enzima que realiza a conversão de angiotensina I em angiotensina II que tem ação vasoconstritora, como também reduzem a degradação da bradicinina que tem ação vasodilatadora. Em contrapartida os BRA atuam bloqueando os receptores específicos de angiotensina II, impedindo sua ação vasoconstritora e promovendo um relaxamento da musculatura lisa e consequentemente promovendo uma vasodilatação (BRUNTON LL, et al., 2019). Em síntese, podemos concluir que os medicamentos antihipertensores corroboram para o risco de queda devido ao fenômeno da hipotensão postural promovido pela vasodilatação gerada pelo seu mecanismo de ação.

Ademais, é de suma importância que se percebeu maior incidência de quedas grave em pacientes do sexo feminino. Tal dado pode ser atribuído tanto ao fato de as mulheres apresentarem maior expectativa de vida, maior cuidado com a saúde e consequentemente maior uso de medicamentos, como também devido a maior incidência de doenças crônicas como a osteoporose e maior prevalência de comportamentos domésticos de risco (AMORIM JSC, et al., 2021).

A maior incidência de osteoporose pode ser atribuída ao fenômeno da menopausa, onde há uma redução dos níveis de estrógeno que atuam de maneira direta nos ossos através de receptores, como de maneira indireta por meio de fatores de crescimento e citocinas. Perante essa alteração hormonal teremos maior ativação dos osteoclastos e consequentemente uma redução de osteoblastos, predominando a reabsorção óssea (RADOMINSKI SC, et al., 2004).

\section{CONSIDERAÇÕES FINAIS}

Este estudo demonstrou a necessidade de uma maior atenção ao se prescrever medicamentos para a população da terceira idade, visto que algumas classes de fármacos apresentam efeitos adversos potencializados neste grupo de pacientes, como por exemplo, o risco de quedas. A literatura em questão se preocupou em avaliar claramente os benzodiazepínicos, diuréticos e anti-hipertensivos no risco de quedas e fraturas decorrentes de quedas em idosos; concluindo que indivíduos do gênero feminino tem uma incidência maior aos riscos de quedas e que pacientes idosos que fazem uso de medicação têm um maior risco de queda/fraturas quando comparado com indivíduos da mesma idade que não utilizam medicamentos. 


\section{REFERÊNCIAS}

1. ALMEIDA ST, et al. Análise de fatores estrínsecos e intrínsecos que predispõem a quedas em idosos. Revista da Associação Médica Brasileira. 2012; 58(4): 427-433.

2. ALVES RLT, et al. Avaliação dos fatores de risco que contribuem para queda em idosos. Revista Brasileira de Geriatria e Gerontologia, 2017; 20(1): 56-66.7.

3. AMORIM JSC, et al. The prevalence of severe falls and associated factors among elderly Brazilians: Results of the 2013 National Health Survey. Ciênc. Saúde Coletiva, 2021; 26(1): 25-30.

4. BARROSO WKS, et al. Diretrizes Brasileiras de Hipertensão Arterial. 2020. Arq. Bras. Cardiol. 2021; 116(3): 516-658.

5. BRUNTON LL, et al. As Bases Farmacológicas da Terapêutica de Goodman \& Gilman. 13nd ed. Porto Alegre: AMGH, 2019; 625p.

6. COUTINHO ESF, SILVA SD. Uso de medicamentos como fator de risco para fratura grave decorrente de queda em idosos. Cadernos de Saúde Pública, 2002;18(5): 1359-1366.

7. DANZA DÁ, et al. Benzodiazepinas y fractura de cadera: estudio de casos y controles. Rev. Méd. Urug., 2015; 31(2): $1-10$.

8. DEGANI GC, et al. Idosos vítimas de trauma: doenças preexistentes, medicamentos em uso no domicílio e índices de trauma. Rev. Bras. Enferm., 2014; 67(5): 759-765.

9. GARCIA BP, et al. Uso de medicamentos asociados al riesgo de caídas enancianos no institucionalizados. Rev. Esc. Enferm. USP, 2018; 52: e03319.

10. GORZONI MR, et al. Medicamentos potencialmente inapropriados para idosos. Revista da Associação Médica Basileira, 2012; 58(4): 442-446.

11. HAMARA A, et al. Correlação entre fratura por queda em idosos e uso prévio de medicamentos. Acta Ortopédica Brasileira, 2007; 15(3): 143-145.

12. LUIZ IC, BRUM AKR. Fatores intrínsecos do risco de queda de idosos no domicílio: estudo descritivo. Online Brazilian Journal Of Nursing, 2018; 16(4): 480-485.

13. MEIRELES AE, et al. Alterações neurológicas fisiológicas ao envelhecimento afetam o sistema mantenedor do equilíbrio. Revista Neurociências, 2010; 18(1): 103-108.

14. MOREIRA FSM, et al. Uso de medicamentos potencialmente inapropriados em idosos institucionalizados: prevalência e fatores associados. Ciênc. Saúde Coletiva, 2020; 25(6): 2073-2082.

15. NASCIMENTO JS, TAVARES DMS. Prevalência e fatores associados a quedas em idosos. Texto \& ContextoEnfermagem, 2016; 25(2): e0360015.

16. NETO AHA, et al. Quedas em idosos institucionalizados: riscos, consequências e antecedentes. Revista Brasileira de Enfermagem, 2017; 70(4): 719-725.

17. OLIVEIRA T, et al. Risco multidimensional de queda em idosos. Revista Brasileira em Promoção da Saúde, 2018; 31(2): 1-9.

18. RADOMINSKI SC, et al. Osteoporose em mulheres na pós-menopausa. Rev. Bras. Reumatol., 2004; 44(60): 426434.

19. REZENDE CP, et al. Queda entre idosos no Brasil e sua relação com o uso de medicamentos: revisão sistemática. Cadernos de Saúde Pública, 2012; 28: 2223-2235.

20. RIBEIRO LCC, et al. Percepção dos idosos sobre as alterações fisiológicas do envelhecimento. Ciência, Cuidado e Saúde, 2009; 8(2): 220-227.

21. RODRIGUES MCS, OLIVEIRA C. Interações medicamentosas e reações adversas a medicamentos na polifarmácia em idosos: uma revisão integrativa. Revista Latino-Americana de Enfermagem, 2016; 24, e2800.

22. SANTOS SSC, et al. Risco de quedas em idosos: revisão integrativa pelo diagnóstico da North American Nursing Diagnosis Association. Rev. Esc. Enferm. USP, 2012; 46(5): :1224-33.

23. SANTOS SSC. Concepções teórico-filosóficas sobre envelhecimento, velhice, idoso e enfermagem gerontogeriátrica. Revista Brasileira de Enfermagem, 2010; 63(6): 1035-9.

24. SILVA P. Farmacologia. 8nd ed. Rio de Janeiro: Guanabara Koogan, 2017; 691p.

25. SILVIA IGP, et al. Perfil Sociodemográfico e clínico de idosos em risco de queda no sul do Brasil. Journal Nursing and Health, 2019; 9(3):e199308.

26. SOUSA LMM, et al. Risco de quedas em idosos residentes na comunidade: revisão sistemática da literatura. Revista Gaúcha de Enfermagem, 2016; 37(4): e55030.

27. SOUZA AR, et al. Contexts and patterns of undue use benzodiazepine among women. Ciência \& Saúde Coletiva, 2013; 8: 1131-1140.

28. TOMAZ SAG, et al. Prevalência de quedas em idosos devido ao uso de benzodiazepínicos e diuréticos. Revista Uningá, 2017; 52(1): 34-39. 